\title{
On a modified degenerate Daehee polynomials and numbers
}

\author{
Jin-Woo Park ${ }^{\mathrm{a}}$, Byung Moon Kim ${ }^{\mathrm{b}}$, Jongkyum Kwon ${ }^{\mathrm{c}, *}$ \\ a Department of Mathematics Education, Daegu University, Gyeongsan-si, Gyeongsangbuk-do, 712-714, Republic of Korea. \\ ${ }^{b}$ Department of Mechanical System Engineering, Dongguk University, 123 Dongdae-ro, Gyungju-si, Gyeongsangbuk-do, 38066, \\ Republic of Korea. \\ ${ }^{c}$ Department of Mathematics Education and RINS, Gyeongsang National University, Jinju, Gyeongsangnamdo, 52828, Republic of \\ Korea.
}

Communicated by Y. J. Cho

\begin{abstract}
The Daehee polynomials and numbers are introduced by Kim and Kim in [D. S. Kim, T. Kim, Appl. Math. Sci. (Ruse), 7 (2013), 5969-5976], and many interesting identities and properties of these polynomials have been found by many researchers. In this paper, we consider the modified degenerated Daehee polynomials and derive some new and interesting identities and properties of those polynomials. (C)2017 All rights reserved.
\end{abstract}

Keywords: p-adic invariant integral on $\mathbb{Z}_{\mathrm{p}}$, degenerate Daehee polynomials, modified degenerate Daehee polynomials. 2010 MSC: $11 \mathrm{~B} 68,11 \mathrm{~S} 40,11 \mathrm{~S} 80$.

\section{Introduction}

Let $p$ be a fixed prime number. Throughout this paper, $\mathbb{Z}_{p}, \mathbb{Q}_{p}$, and $\mathbb{C}_{p}$ will respectively denote the ring of $p$-adic rational integers, the field of $p$-adic rational numbers, and the completions of algebraic closure of $\mathbb{Q}_{p}$. The $p$-adic norm $|\cdot|_{p}$ is defined normally as $|p|_{p}=\frac{1}{p}$.

Let $\mathrm{C}\left(\mathbb{Z}_{p}\right)$ be the space of continuous functions on $\mathbb{Z}_{p}$. For $f \in C\left(\mathbb{Z}_{p}\right)$, the $p$-adic invariant integral on $\mathbb{Z}_{\mathrm{p}}$ is defined by Kim as follows [7, 8].

$$
I_{0}(f)=\int_{\mathbb{Z}_{p}} f(x) d \mu_{0}(x)=\lim _{n \rightarrow \infty} \frac{1}{p^{n}} \sum_{x=0}^{p^{n}-1} f(x) .
$$

If we put $f_{n}(x)=f(x+n)$, then, by (1.1), we can derive the following very useful integral identity;

$$
I_{0}\left(f_{n}\right)-I_{0}(f)=\sum_{l=0}^{n-1} f^{\prime}(l)
$$

\footnotetext{
*Corresponding author

Email addresses: a0417001@knu.ac.kr (Jin-Woo Park), kbm713@dongguk.ac.kr (Byung Moon Kim), mathkjk26@gnu.ac.kr (Jongkyum Kwon)

doi:10.22436/jnsa.010.03.21
} 
In particular, if $n=1$, then

$$
I_{0}\left(f_{1}\right)-I_{0}(f)=f^{\prime}(0) .
$$

The Stirling numbers of the first kind are given by

$$
(x)_{n}=x(x-1) \cdots(x-n+1)=\sum_{l=0}^{n} S_{1}(n, l) x^{l}(x \geqslant 0),
$$

and the Stirling numbers of the second kind are defined by the generating function to be

$$
\left(e^{t}-1\right)^{n}=n ! \sum_{l=n}^{\infty} S_{2}(l, n) \frac{t^{l}}{l !}
$$

(see $[2,25])$. Note that

$$
(\log (x+1))^{n}=n ! \sum_{l=n}^{\infty} S_{1}(l, n) \frac{x^{l}}{l !},(n \geqslant 0)
$$

(see $[2,25])$.

As is well-known, Bernoulli polynomials of order $r$ are defined by the generating function (see [3-6, 9-24])

$$
\left(\frac{t}{e^{t}-1}\right)^{r} e^{x t}=\sum_{n=0}^{\infty} B_{n}^{(r)}(x) \frac{t^{n}}{n !}
$$

In the special case, $x=0, \mathrm{~B}_{\mathrm{n}}^{(\mathrm{r})}=\mathrm{B}_{\mathrm{n}}^{(\mathrm{r})}(0)$ are called the Bernoulli numbers of order $\mathrm{r}$.

From (1.1), we note that

$$
\sum_{n=0}^{\infty} B_{n}^{(r)}(x) \frac{t^{n}}{n !}=\left(\frac{t}{e^{t}-1}\right)^{r} e^{x t}=e^{x t} \int_{\mathbb{Z}_{p}} \cdots \int_{\mathbb{Z}_{p}} e^{\left(x_{1}+\cdots+x_{r}\right) t} d \mu_{0}\left(x_{1}\right) \cdots d \mu_{0}\left(x_{r}\right),
$$

and by (1.4), we have

$$
B_{n}^{(r)}(x)=\int_{\mathbb{Z}_{p}}\left(x_{1}+\cdots+x_{r}+x\right)^{n} d \mu_{0}\left(x_{1}\right) \cdots d \mu_{0}\left(x_{r}\right),(n \geqslant 0)
$$

(see [3-6, 9-24]).

In $[6,10,17-19,26,27]$, authors defined the Daehee polynomials as follows,

$$
\sum_{n=0}^{\infty} D_{n}(x) \frac{t^{n}}{n !}=\frac{\log (1+t)}{t}(1+t)^{x}
$$

and, in $[4,5]$, authors defined the modified degenerate Bernoulli polynomials of order $r$ as follows:

$$
\sum_{n=0}^{\infty} \beta_{n, \lambda}^{(r)}(x) \frac{t^{n}}{n !}=\left(\frac{t}{(1+\lambda)^{\frac{t}{\lambda}}-1}\right)^{r}(1+\lambda)^{\frac{t}{\lambda} x} .
$$

Recently, Daehee numbers and polynomials are introduced by Kim and Kim in [10], and by many mathematicians, which are generalized and obtained many new and interesting properties (see [1, 3, $6,10,12-20,22-24,26,27]$. In this paper, we consider the modified degenerate Daehee polynomials and numbers by using the p-adic invariant integral, and derive some new and interesting identities and properties of those polynomials. 


\section{Modified degenerate Daehee polynomials and numbers}

From now on, we assume that $t \in \mathbb{C}$ with $|t|_{p}<p^{-\frac{1}{p-1}}$ and $\lambda \in \mathbb{Z}_{\mathfrak{p}}$.

The modified degenerate Daehee polynomials are defined by the generating function to be

$$
\frac{\frac{\log (1+\lambda)}{\lambda} \log (1+t)}{(1+\lambda)^{\frac{1}{\lambda} \log (1+t)}-1}(1+\lambda)^{\frac{x}{\lambda} \log (1+t)}=\sum_{n=0}^{\infty} M_{n, \lambda}(x) \frac{t^{n}}{n !} .
$$

In the special case, $x=0, M D_{n, \lambda}=M_{n, \lambda}(0)$ are called modified degenerate Daehee numbers.

Note that

$$
\lim _{\lambda \rightarrow 0} \frac{\frac{\log (1+\lambda)}{\lambda} \log (1+t)}{(1+\lambda)^{\frac{1}{\lambda} \log (1+t)}-1}(1+\lambda)^{\frac{x}{\lambda} \log (1+t)}=\frac{\log (1+t)}{t}(1+t)^{x}=\sum_{n=0}^{\infty} D_{n}(x) \frac{t^{n}}{n !} .
$$

Since

$$
\begin{aligned}
(1+\lambda)^{\frac{x+y}{\lambda} \log (1+t)} & =e^{\log (1+\lambda)^{\frac{x+y}{\lambda} \log (1+t)}} \\
& =\sum_{n=0}^{\infty}\left(\frac{\log (1+\lambda)}{\lambda}\right)^{n}(x+y)^{n}(\log (1+t))^{n} \frac{1}{n !} \\
& =\sum_{n=0}^{\infty}\left(\frac{\log (1+\lambda)}{\lambda}\right)^{n}(x+y)^{n} \frac{1}{n !} n ! \sum_{l=n}^{\infty} S_{1}(l, n) \frac{t^{l}}{l !} \\
& =\sum_{n=0}^{\infty} \sum_{m=0}^{n}\left(\frac{\log (1+\lambda)}{\lambda}\right)^{m}(x+y)^{m} S_{1}(n, m) \frac{t^{n}}{n !}
\end{aligned}
$$

and

$$
\begin{aligned}
& \sum_{n=0}^{\infty} M D_{n, \lambda}(x) \frac{t^{n}}{n !}=\frac{\frac{\log (1+\lambda)}{\lambda} \log (1+t)}{(1+\lambda)^{\frac{1}{\lambda} \log (1+t)}-1}(1+\lambda)^{\frac{x}{\lambda} \log (1+t)} \\
& =\left(\sum_{n=0}^{\infty} M D_{n, \lambda} \frac{t^{n}}{n !}\right)\left(\sum_{m=0}^{\infty} \sum_{l=0}^{m}\left(\frac{\log (1+\lambda)}{\lambda}\right)^{l} S_{1}(m, l) x^{l} \frac{t^{m}}{m !}\right) \\
& =\sum_{n=0}^{\infty} \sum_{m=0}^{n} \sum_{l=0}^{m}\left(\begin{array}{c}
n \\
m
\end{array}\right)\left(\frac{\log (1+\lambda)}{\lambda}\right)^{l} S_{1}(m, l) x^{l} M D_{n-m, \lambda} \frac{t^{n}}{n !},
\end{aligned}
$$

by (2.2) and (2.3), we obtain the following theorem.

Theorem 2.1. For each nonnegative integer $n$, we have

$$
\operatorname{MD}_{n, \lambda}(x)=\sum_{m=0}^{n} \sum_{l=0}^{m}\left(\begin{array}{l}
n \\
m
\end{array}\right)\left(\frac{\log (1+\lambda)}{\lambda}\right)^{l} S_{1}(m, l) M D_{n-m, \lambda} x^{l} .
$$

Note that, by (1.3), we have

$$
\int_{\mathbb{Z}_{p}}(1+\lambda)^{\frac{x+y}{\lambda} \log (1+t)} d \mu_{0}(y)=\frac{\frac{\log (1+\lambda)}{\lambda} \log (1+t)}{(1+\lambda)^{\frac{1}{\lambda} \log (1+t)}-1}(1+\lambda)^{\frac{x}{\lambda} \log (1+t)}=\sum_{n=0}^{\infty} M_{n, \lambda}(x) \frac{t^{n}}{n !},
$$

and, by (2.2) and (1.5),

$$
\begin{aligned}
\int_{\mathbb{Z}_{p}}(1+\lambda)^{\frac{x+y}{\lambda} \log (1+t)} d \mu_{0}(y) & =\sum_{n=0}^{\infty} \int_{\mathbb{Z}_{p}} \sum_{m=0}^{n}\left(\frac{\log (1+\lambda)}{\lambda}\right)^{m}(x+y)^{m} S_{1}(n, m) d \mu_{0}(y) \frac{t^{n}}{n !} \\
& =\sum_{n=0}^{\infty} \sum_{m=0}^{n}\left(\frac{\log (1+\lambda)}{\lambda}\right)^{m} S_{1}(n, m) B_{m}(x) \frac{t^{n}}{n !} .
\end{aligned}
$$

Therefore, by (2.4) and (2.5), we obtain the following theorem. 
Theorem 2.2. For each nonnegative integer $n$, we have

and

$$
\sum_{n=0}^{\infty} M D_{n, \lambda}(x) \frac{t^{n}}{n !}=\int_{\mathbb{Z}_{p}}(1+\lambda)^{\frac{x+y}{\lambda} \log (1+t)} d \mu_{0}(y)
$$

$$
\operatorname{MD}_{n, \lambda}(x)=\sum_{m=0}^{n}\left(\frac{\log (1+\lambda)}{\lambda}\right)^{m} S_{1}(n, m) B_{m}(x) .
$$

By replacing $t$ by $e^{t}-1$ in (2.1), we have

$$
\begin{aligned}
\frac{\frac{\log (1+\lambda)}{\lambda} t}{(1+\lambda)^{\frac{t}{\lambda}}-1}(1+\lambda)^{\frac{t}{\lambda} x} & =\sum_{n=0}^{\infty} \operatorname{MD}_{n, \lambda}(x) \frac{1}{n !}\left(e^{t}-1\right)^{n} \\
& =\sum_{n=0}^{\infty} M_{n, \lambda}(x) \frac{1}{n !} n ! \sum_{l=n}^{\infty} S_{2}(l, n) \frac{t^{l}}{l !} \\
& =\sum_{n=0}^{\infty} \sum_{m=0}^{n} M_{m, \lambda}(x) S_{2}(n, m) \frac{t^{n}}{n !}
\end{aligned}
$$

and

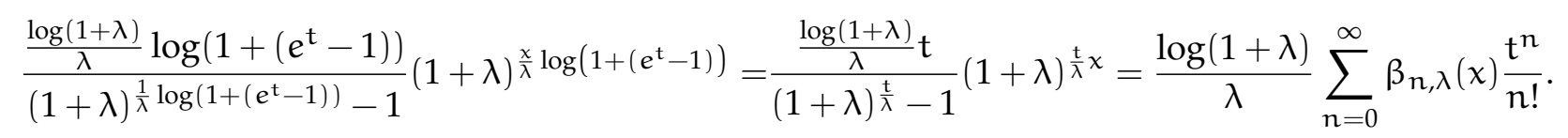

By (2.6) and (2.7), we obtain the following corollary.

Corollary 2.3. For each nonnegative integer $n$,

$$
\beta_{n, \lambda}(x)=\frac{\lambda}{\log (1+\lambda)} \sum_{m=0}^{n} \operatorname{MD}_{m, \lambda}(x) S_{2}(n, m) .
$$

By (1.3), we note that

$$
\begin{aligned}
\frac{\log (1+\lambda)}{\lambda} \log (1+t) & =\int_{\mathbb{Z}_{p}}(1+\lambda)^{\frac{y+1}{\lambda}} \log (1+t) d \mu_{0}(y)-\int_{\mathbb{Z}_{p}}(1+\lambda)^{\frac{y}{\lambda} \log (1+t)} d \mu_{0}(y) \\
& =\sum_{n=0}^{\infty} M D_{n, \lambda}(1) \frac{t^{n}}{n !}-\sum_{n=0}^{\infty} M D_{n, \lambda} \frac{t^{n}}{n !} \\
& =\sum_{n=0}^{\infty}\left(M D_{n, \lambda}(1)-M D_{n, \lambda}\right) \frac{t^{n}}{n !}
\end{aligned}
$$

Since

$$
\log (1+t)=\sum_{n=1}^{\infty}(-1)^{n-1} \frac{t^{n}}{n}
$$

by (2.8) and (2.9), we obtain the following theorem.

Theorem 2.4. For each nonnegative integer $n$, we have

$$
\mathrm{MD}_{0, \lambda}=1, \mathrm{MD}_{n, \lambda}(1)-\mathrm{MD}_{n, \lambda}=(-1)^{n-1} \frac{\log (1+\lambda)}{\lambda},(n \geqslant 1) .
$$


For each $n \in \mathbb{N}$, by (1.2) and (2.9), we have

$$
\begin{aligned}
& \int_{\mathbb{Z}_{p}}(1+\lambda)^{\frac{y+n}{\lambda}} \log (1+t) d \mu_{0}(y)-\int_{\mathbb{Z}_{p}}(1+\lambda)^{\frac{y}{\lambda} \log (1+t)} d \mu_{0}(y) \\
& =\sum_{a=0}^{n-1} \frac{\log (1+\lambda)}{\lambda} \log (1+t)(1+\lambda)^{\frac{a}{\lambda} \log (1+t)} \\
& =\sum_{k=0}^{\infty}\left(\sum_{l=0}^{k} \sum_{m=0}^{l} \sum_{a=0}^{n-1}(-1)^{k-l}\left(\frac{\log (1+\lambda)}{\lambda}\right)^{m+1} a^{m} S_{1}(l, m)\left(\begin{array}{l}
k \\
l
\end{array}\right) \frac{k+1}{k-l+1}\right) \frac{t^{k+1}}{(k+1) !}
\end{aligned}
$$

and

$$
\int_{\mathbb{Z}_{\mathrm{p}}}(1+\lambda)^{\frac{y+n}{\lambda} \log (1+\mathrm{t})} \mathrm{d} \mu_{0}(\mathrm{y})-\int_{\mathbb{Z}_{\mathrm{p}}}(1+\lambda)^{\frac{y}{\lambda} \log (1+\mathrm{t})} \mathrm{d} \mu_{0}(\mathrm{y})=\sum_{\mathrm{k}=0}^{\infty}\left(M D_{k, \lambda}(\mathrm{n})-M D_{k, \lambda}\right) \frac{\mathrm{t}^{\mathrm{k}}}{\mathrm{k} !}
$$

Hence, by (2.10) and (2.11), we obtain the following theorem.

Theorem 2.5. For each nonnegative integer $\mathrm{n}$ and each positive integer $\mathrm{k}$, we have

$$
\mathrm{MD}_{0, \lambda}(\mathrm{n})=\mathrm{MD}_{0, \lambda}=1,
$$

and

$$
M D_{k+1, \lambda}(n)-M D_{k+1, \lambda}=\sum_{l=0}^{k} \sum_{m=0}^{l} \sum_{a=0}^{n-1}(-1)^{k-l}\left(\frac{\log (1+\lambda)}{\lambda}\right)^{m+1} a^{m} S_{1}(l, m)\left(\begin{array}{l}
k \\
l
\end{array}\right) \frac{k+1}{k-l+1} .
$$

From now on, we consider the modified degenerate Daehee polynomials of order $r$ defined by the generating function as follows:

$$
\sum_{n=0}^{\infty} M D_{n, \lambda}^{(r)}(x) \frac{t^{n}}{n !}=\int_{\mathbb{Z}_{p}} \cdots \int_{\mathbb{Z}_{p}}(1+\lambda)^{\frac{x_{1}+\cdots+x_{r}+x}{\lambda}} \log (1+t) d \mu_{0}\left(x_{1}\right) \cdots d \mu_{0}\left(x_{r}\right) .
$$

When $x=0, M D_{n, \lambda}^{(r)}=M D_{n, \lambda}^{(r)}(0)$ are called modified degenerate Changhee numbers of order $r$.

Note that, by (1.1) and (2.2),

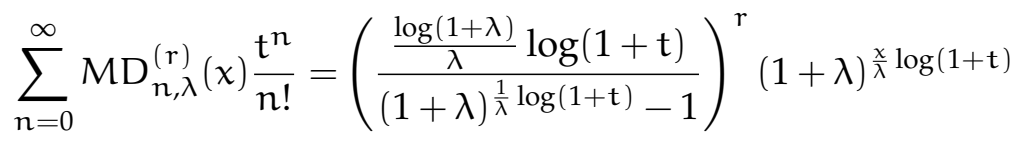

$$
\begin{aligned}
& =\left(\sum_{n=0}^{\infty} \operatorname{MDn}, \lambda \frac{t^{n}}{n !}\right)^{r}\left(\sum_{n=0}^{\infty} \sum_{m=0}^{n}\left(\frac{\log (1+\lambda)}{\lambda}\right)^{m} x^{m} S_{1}(n, m) \frac{t^{n}}{n !}\right) \\
& =\left(\sum_{n=0}^{\infty} \sum_{\substack{n_{1} \ldots n_{r} \geqslant 0 \\
n_{1}+\cdots+n_{r}=n}}^{\infty} M_{n_{1}, \lambda} \cdots M D_{n_{r}, \lambda} \frac{t^{n_{1}}}{n_{1} !} \cdots \frac{t^{n_{r}}}{n_{r} !}\right) \\
& \times\left(\sum_{n=0}^{\infty} \sum_{m=0}^{n}\left(\frac{\log (1+\lambda)}{\lambda}\right)^{m} x^{m} S_{1}(n, m) \frac{t^{n}}{n !}\right) \\
& =\sum_{n=0}^{\infty}\left(\sum_{m=0}^{n} \sum_{\substack{n_{1}, \ldots, n_{r} \geqslant 0 \\
n_{1}+\cdots+n_{r}=m}} \sum_{k=0}^{n-m}\left(\begin{array}{c}
m \\
n_{1}, \cdots, n_{r}
\end{array}\right)\left(\begin{array}{l}
n \\
m
\end{array}\right) M D_{n_{1}, \lambda} \cdots M D_{n_{r}, \lambda}\right. \\
& \left.\times\left(\frac{\log (1+\lambda)}{\lambda}\right)^{k} x^{k} S_{1}(n-m, k)\right) \frac{t^{n}}{n !},
\end{aligned}
$$

where $\left(\begin{array}{c}m \\ n_{1}, \ldots, n_{r}\end{array}\right)$ are the multinomial coefficients. 
In addition, by (1.1) and (2.2), we have

$$
\begin{aligned}
\int_{\mathbb{Z}_{\mathfrak{p}}} \cdots \int_{\mathbb{Z}_{\mathfrak{p}}} & (1+\lambda)^{\frac{x_{1}+\cdots+x_{r}+x}{\lambda} \log (1+t)} \mathrm{d} \mu_{0}\left(x_{1}\right) \cdots d \mu_{0}\left(x_{r}\right) \\
= & \sum_{n=0}^{\infty} \sum_{m=0}^{n}\left(\frac{\log (1+\lambda)}{\lambda}\right)^{m} S_{1}(n, m) \int_{\mathbb{Z}_{p}} \cdots \int_{\mathbb{Z}_{p}}\left(x_{1}+\cdots+x_{r}+x\right)^{m} d \mu_{0}\left(x_{1}\right) \cdots d \mu_{0}\left(x_{r}\right) \frac{t^{n}}{n !} \\
= & \sum_{n=0}^{\infty} \sum_{m=0}^{n}\left(\frac{\log (1+\lambda)}{\lambda}\right)^{m} S_{1}(n, m) B_{m}^{(r)}(x) \frac{t^{n}}{n !} .
\end{aligned}
$$

By (2.12), (2.13), and (2.14), we obtain the following theorem.

Theorem 2.6. For each nonnegative integer $n$, we have

$$
M D_{n, \lambda}^{(r)}(x)=\sum_{m=0}^{n} \sum_{\substack{n_{1}, \ldots, n_{r} \geqslant 0 \\
n_{1}+\cdots+n_{r}=m}} \sum_{k=0}^{n-m}\left(\begin{array}{c}
m \\
n_{1}, \cdots, n_{r}
\end{array}\right)\left(\begin{array}{c}
n \\
m
\end{array}\right) M D_{n_{1}, \lambda} \cdots M D_{n_{r}, \lambda} \times\left(\frac{\log (1+\lambda)}{\lambda}\right)^{k} S_{1}(n-m, k) x^{k},
$$

and

$$
\operatorname{MD}_{n, \lambda}^{(r)}(x)=\sum_{m=0}^{n}\left(\frac{\log (1+\lambda)}{\lambda}\right)^{m} S_{1}(n, m) B_{m}^{(r)}(x)
$$

By replacing $t$ by $e^{t}-1$ in (2.13), we get

$$
\begin{aligned}
\left(\frac{\frac{\log (1+\lambda)}{\lambda} t}{(1+\lambda)^{\frac{t}{\lambda}}-1}\right)^{r}(1+\lambda)^{\frac{t}{\lambda} x} & =\sum_{n=0}^{\infty} M D_{n, \lambda}^{(r)}(x) \frac{1}{n !}\left(e^{t}-1\right)^{m}=\sum_{n=0}^{\infty} M D_{n, \lambda}^{(r)}(x) \frac{1}{n !} n ! \sum_{l=n}^{\infty} S_{2}(l, n) \frac{t^{l}}{l !} \\
& =\sum_{n=0}^{\infty} \sum_{m=0}^{n} M D_{m, \lambda}^{(r)}(x) S_{2}(n, m) \frac{t^{n}}{n !}
\end{aligned}
$$

and

$$
\begin{aligned}
& \left(\frac{\frac{\log (1+\lambda)}{\lambda} \log \left(1+\left(e^{t}-1\right)\right)}{(1+\lambda)^{\frac{1}{\lambda} \log \left(1+\left(e^{t}-1\right)\right)}-1}\right)^{r}(1+\lambda)^{\frac{x}{\lambda} \log \left(1+\left(e^{t}-1\right)\right)}=\left(\frac{\frac{\log (1+\lambda)}{\lambda} t}{(1+\lambda)^{\frac{t}{\lambda}}-1}\right)^{r}(1+\lambda)^{\frac{x t}{\lambda}} \\
& =\sum_{n=0}^{\infty}\left(\frac{\log (1+\lambda)}{\lambda}\right)^{r} \beta_{n, \lambda}^{(r)}(x) \frac{t^{n}}{n !} \text {. }
\end{aligned}
$$

By (2.15) and (2.16), we obtain the following theorem.

Theorem 2.7. For each $\mathrm{n} \geqslant 0$, we have

$$
\beta_{n, \lambda}^{(r)}(x)=\left(\frac{\lambda}{\log (1+\lambda)}\right)^{r} \sum_{m=0}^{n} M_{m, \lambda}^{(r)}(x) S_{2}(n, m) .
$$

By (2.13), we observe that

$$
\begin{aligned}
& \sum_{n=0}^{\infty}\left(M D_{n, \lambda}^{(r)}(x+1)-M D_{n, \lambda}^{(r)}(x)\right) \frac{t^{n}}{n !} \\
& =\left(\frac{\frac{\log (1+\lambda)}{\lambda} \log (1+t)}{(1+\lambda)^{\frac{1}{\lambda} \log (1+t)}-1}\right)^{r}(1+\lambda)^{\frac{x+1}{\lambda} \log (1+t)}-\left(\frac{\frac{\log (1+\lambda)}{\lambda} \log (1+t)}{(1+\lambda)^{\frac{1}{\lambda} \log (1+t)}-1}\right)^{r}(1+\lambda)^{\frac{x}{\lambda} \log (1+t)}
\end{aligned}
$$




$$
\begin{aligned}
& =\left(\frac{\frac{\log (1+\lambda)}{\lambda} \log (1+t)}{(1+\lambda)^{\frac{1}{\lambda} \log (1+t)}-1}\right)^{r-1}(1+\lambda)^{\frac{x}{\lambda} \log (1+t)} \\
& =\sum_{n=0}^{\infty} M D_{n, \lambda}^{(r-1)}(x) \frac{t^{n}}{n !} .
\end{aligned}
$$

Therefore, by (2.17), we obtain the following theorem.

Theorem 2.8. For each $\mathrm{n} \geqslant 0$ and $\mathrm{r} \in \mathbb{N}$, we have

$$
M D_{n, \lambda}^{(r)}(x+1)-M D_{n, \lambda}^{(r)}(x)=M_{n, \lambda}^{(r-1)}(x) .
$$

Now, we consider the modified degenerate Daehee polynomials of the second kind defined by the generating function as follows:

$$
\sum_{n=0}^{\infty} \widehat{M D}_{n, \lambda}(x) \frac{t^{n}}{n !}=\int_{\mathbb{Z}_{p}}(1+\lambda)^{\frac{x-y}{\lambda} \log (1+t)} d \mu_{0}(y) .
$$

By (1.1), we have

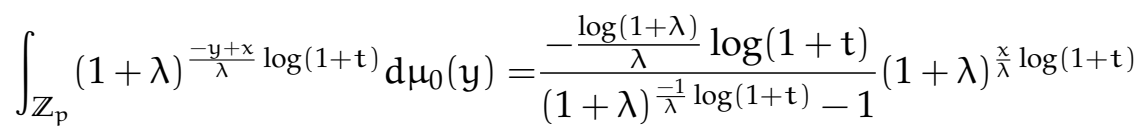

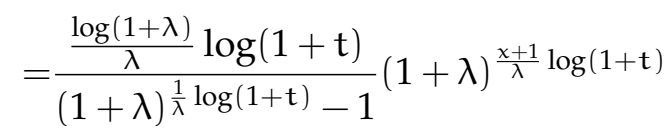

$$
\begin{aligned}
& =\sum_{n=0}^{\infty} M_{n, \lambda}(x+1) \frac{t^{n}}{n !} \text {, }
\end{aligned}
$$

and, by (2.2),

$$
\begin{aligned}
\int_{\mathbb{Z}_{p}}(1+\lambda)^{\frac{-y+x}{\lambda}} \log (1+t) d \mu_{0}(y) & =\sum_{n=0}^{\infty} \sum_{m=0}^{n}\left(\frac{\log (1+\lambda)}{\lambda}\right)^{m} S_{1}(n, m) \int_{\mathbb{Z}_{p}}(x-y)^{m} d \mu_{0}(y) \frac{t^{n}}{n !} \\
& =\sum_{n=0}^{\infty} \sum_{m=0}^{n}\left(\frac{\log (1+\lambda)}{\lambda}\right)^{m} S_{1}(n, m)(-1)^{m} B_{m}(-x) \frac{t^{n}}{n !} .
\end{aligned}
$$

Thus, by (2.18), (2.19), and (2.20), we have the following theorem.

Theorem 2.9. For each $\mathrm{n} \geqslant 0$, we have

$$
\widehat{M D}_{n, \lambda}(x)=\operatorname{MD}_{n, \lambda}(x+1),
$$

and

$$
\widehat{M D}_{n, \lambda}(x)=\sum_{m=0}^{n}\left(\frac{\log (1+\lambda)}{\lambda}\right)^{m} S_{1}(n, m)(-1)^{m} B_{m}(-x) .
$$

\section{References}

[1] A. Bayad, Special values of Lerch zeta function and their Fourier expansions, Adv. Stud. Contemp. Math. (Kyungshang), 21 (2011), 1-4. 1

[2] L. Comtet, Advanced combinatorics, The art of finite and infinite expansions, Revised and enlarged edition, D. Reidel Publishing Co., Dordrecht, (1974). 1

[3] D. V. Dolgy, D. S. Kim, T. Kim, T. Mansour, Barnes-type Daehee with $\lambda$-parameter and degenerate Euler mixed-type polynomials, J. Inequal. Appl., 2015 (2015), 13 pages. 1, 1, 1 
[4] D. V. Dolgy, T. Kim, H. I. Kwon, J. J. Seo, On the modified degenerate Bernoulli polynomials, Adv. Stud. Contemp. Math. (Kyungshang), 26 (2016), 1-9. 1

[5] D. V. Dolgy, T. Kim, J. J. Seo, On the symmetric identities of modified degenerate Bernoulli polynomials, Proc. Jangjeon Math. Soc., 19 (2016), 301-308. 1

[6] B. S. El-Desouky, A. Mustafa, New results on higher-order Daehee and Bernoulli numbers and polynomials, Adv. Difference Equ., 2016 (2016), 21 pages. 1, 1, 1

[7] T. Kim, On a q-analogue of the p-adic log gamma functions and related integrals, J. Number Theory, 76 (1999), $320-329$. 1

[8] T. Kim, q-Volkenborn integration, Russ. J. Math. Phys., 9 (2002), 288-299. 1

[9] T. Kim, q-Bernoulli numbers and polynomials associated with Gaussian binomial coefficients, Russ. J. Math. Phys., 15 (2008), 51-57. 1, 1

[10] D. S. Kim, T. Kim, Daehee numbers and polynomials, Appl. Math. Sci. (Ruse), 7 (2013), 5969-5976. 1, 1

[11] D. S. Kim, T. Kim, Barnes-type Daehee of the second kind and poly-Cauchy of the second kind mixed-type polynomials, J. Inequal. Appl., 2014 (2014), 19 pages.

[12] D. S. Kim, T. Kim, Identities arising from higher-order Daehee polynomial bases, Open Math., 13 (2015), 196-208. 1

[13] D. S. Kim, T. Kim, On degenerate Bell numbers and polynomials, Rev. R. Acad. Cienc. Exactas Fs. Nat., Ser. A Mat., 2016 (2016), 1-12.

[14] D. S. Kim, T. Kim, T. Komatsu, S.-H. Lee, Barnes-type Daehee of the first kind and poly-Cauchy of the first kind mixed-type polynomials, Adv. Difference Equ., 2014 (2014), 22 pages.

[15] T. Kim, D. S. Kim, T. Komatsu, S.-H. Lee, Higher-order Daehee of the second kind and poly-Cauchy of the second kind mixed-type polynomials, J. Nonlinear Convex Anal., 16 (2015), 1993-2015.

[16] D. S. Kim, T. Kim, T. Komatsu, J.-J. Seo, Barnes-type Daehee polynomials, Adv. Difference Equ., 2014 (2014), 6 pages.

[17] D. S. Kim, T. Kim, S.-H. Lee, Higher-order Daehee of the first kind and poly-Cauchy of the first kind mixed type polynomials, J. Comput. Anal. Appl., 18 (2015), 699-714. 1

[18] D. S. Kim, T. Kim, S.-H. Lee, J.-J. Seo, Higher-order Daehee numbers and polynomials, Int. J. Math. Anal. (Ruse), 8 (2014), 273-283.

[19] D. S. Kim, T. Kim, J.-J. Seo, Higher-order Daehee polynomials of the first kind with umbral calculus, Adv. Stud. Contemp. Math. (Kyungshang), 24 (2016), 5-18. 1

[20] T. Kim, Y. Simsek, Analytic continuation of the multiple Daehee q-l-functions associated with Daehee numbers, Russ. J. Math. Phys., 15 (2008), 58-65. 1

[21] V. Kurt, Some relation between the Bernstein polynomials and second kind Bernoulli polynomials, Adv. Stud. Contemp. Math. (Kyungshang), 23 (2013), 43-48.

[22] E.-J. Moon, J.-W. Park, S.-H. Rim, A note on the generalized q-Daehee numbers of higher order, Proc. Jangjeon Math. Soc., 17 (2014), 557-565. 1

[23] H. Ozden, I. N. Cangul, Y. Simsek, Remarks on q-Bernoulli numbers associated with Daehee numbers, Adv. Stud. Contemp. Math. (Kyungshang), 18 (2009), 41-48.

[24] J.-W. Park, On the q-analogue of $\lambda$-Daehee polynomials, J. Comput. Anal. Appl., 19 (2015), 966-974. 1, 1, 1

[25] S. Roman, The umbral calculus, Academic Press, New York, (2005). 1

[26] J.-J. Seo, T. Kim, Some identities of symmetry for Daehee polynomials arising from p-adic invariant integral on $\mathbb{Z}_{\mathrm{p}}$, Proc. Jangjeon Math. Soc., 19 (2016), 285-292. 1, 1

[27] J.-J. Seo, S.-H. Rim, T. Kim, S.-H. Lee, Sums products of generalized Daehee numbers, Proc. Jangjeon Math. Soc., 17 (2014), 1-9. 1, 1 